\title{
CIRURGIA DE OSTEOMA DE SEIO FRONTAL
}

\author{
Lisete Pessoa de Oliveira Fobe', Erich Christiano de Melo², \\ Luiz Fernando Cannone', Jean-Luc Fobe ${ }^{4}$
}

RESUMO - Os osteomas do seio frontal correspondem a 57\% dos osteomas dos seios paranasais, com incidência variando de $0,01 \%$ a 3\%. A remoção cirúrgica nos osteomas frontais é indicada nos pacientes sintomáticos. Nos pacientes assintomáticos pode-se adotar a conduta conservadora ou cirúrgica em todos os pacientes independente da sua localização ou extensão. Cinco pacientes com diagnóstico de osteoma de seio frontal foram operados entre 1995 e 1999. A idade média foi 38,4 anos (extremos de 12 a 55 anos), sendo 3 homens e 2 mulheres. 0 período de sintomatologia variou de 6 meses a 3 anos com média de 10,5 meses. Quatro pacientes apresentaram cefaléia. Um paciente apresentou epistaxe. Os exames complementares realizados foram: radiografia simples e tomografia computadorizada de seios paranasais com cortes axiais e coronais. Em dois pacientes o diâmetro do osteoma foi maior que $3 \mathrm{~cm}$, e menor que $3 \mathrm{~cm}$ em três. A decisão da técnica cirúrgica entre coronal e supraciliar foi estética, reservando-se a abordagem supraciliar para um paciente com calvície, apesar do tumor ser volumoso com extensão para seio etmoidal. Nenhuma dificuldade técnica intraoperatória foi atribuída à escolha da abordagem. 0 óstio nasofrontal não foi obstruído no intra-operatório. 0 seguimento pós-operatório mínimo foi de dois anos. Em todos os casos a remoção foi total sem recidiva ou resíduos tumorais. Os sintomas clínicos, achados radiológicos e abordagens cirúrgicas são discutidos. Não ocorreram complicações pós-operatórias.

PALAVRAS-CHAVE: osteoma, seio frontal, seios paranasais.

\begin{abstract}
Surgery of frontal sinus osteoma
ABSTRACT - Frontal sinus osteomas are $57 \%$ of all paranasal sinus osteomas, with an incidence of 00.1 to $3 \%$. Surgical removal of the frontal sinus osteomas is done in symptomatic patients. Asymptomatic patients can be managed conservatively or submitted to surgery in spite of its location or extension. Five patients having the diagnosis of frontal sinus osteoma were operated on between 1995 and 1999 . Medium age was 38.4 years (from 12 to 55 years), 3 male and 2 female. Symptoms occurred from 6 months to 3 years, average of 10.5 months. Four patients had previous headache and one had epistaxis. All patients had standard radiological exams and computed tomography with coronal and axial studies of paranasal sinus. In two patients the diameter of the osteoma was larger than $3 \mathrm{~cm}$ and in three smaller than $3 \mathrm{~cm}$. The choice between coronal and supraciliar approach was made according to esthetics, supraciliar approach was made in only one bald patient even with the tumor being large and extending to ethmoidal sinus. Any intra operative difficulty was related to the choice of the approach. Naso-frontal ostium was not obstructed in intra operative course. Minimal postoperative follow up was of two years. Osteomas were radically removed in all patients with no recurrence or residual tumor. Clinical findings, radiological exams and surgical approaches are discussed. No postoperative complications occurred.
\end{abstract}

KEY WORDS: frontal sinus, osteoma, paranasal sinus.

Os osteomas dos seios paranasais são tumores histologicamente benignos que, apesar de infrequentes, não são raros. São lesões ósseas encapsuladas com potencial de crescimento ilimitado. Deslocam estruturas adjacentes e estão usualmente aderidos ao osso frontal do qual têm origem ${ }^{1}$. Os osteomas do seio frontal correspondem a $57 \%$ dos osteomas dos seios paranasais ${ }^{1}$, com uma incidên- cia variando de $0,01 \%$ a 3\% $\%^{2-4}$. A remoção cirúrgica destes tumores é geralmente indicada nos pacientes sintomáticos ${ }^{3,5}$. Nos pacientes assintomáticos pode-se adotar a conduta conservadora ou cirúrgica independentemente da sua localização ou extensão $0^{6,7}$. Nos pacientes assintomáticos foram propostos critérios para a indicação do procedimento cirúrgico, como extensão dos seus limites para além

Otorrinolaringologia e Neurocirurgia do Hospital São Luiz, São Paulo SP, Brasil: ${ }^{1}$ Otorrinolaringologista, Doutora pela Faculdade de Medicina da Universidade de São Paulo; ${ }^{2}$ Médico Residente de Otorrinolaringologia do Hospital do Servidor Público; ${ }^{3}$ Médico Neurocirurgião, Assistente da Neurocirurgia da Santa Casa de São Paulo; ${ }^{4}$ Médico Neurocirurgião.

Recebido 5 Julho 2001, recebido na forma final 1 Outubro 2001. Aceito 11 Outubro 2001. 
do seio frontal, crescimento progressivo ${ }^{8}$, proximidade ao ducto nasofrontal ou se ocupa mais de $50 \%$ do diâmetro do seio frontal 5 .

Cinco pacientes sintomáticos foram operados de osteoma de seio frontal com um seguimento mínimo de 2 anos. As manifestações clínicas, indicações, exames radiológicos, técnica cirúrgica empregada e evolução pós-operatória são apresentados.

\section{CASUÍSTICA}

Cinco pacientes com diagnóstico de osteoma sintomáticos de seio frontal foram operados. O seguimento pósoperatório médio foi de 4 anos (extremos entre de 2 e 6 anos). A idade média foi de 38,4 anos (extremos de 12 a 55 anos), sendo 3 homens e 2 mulheres. O período de evo- lução da sintomatologia variou de 6 meses a 3 anos com média de 10,5 meses. O motivo da consulta inicial foi principalmente cefaléia frontal crônica. Em dois pacientes existia sinusopatia maxilar, não sendo correlacionada fisiopatologicamente como causada pelo osteoma. Em uma paciente o osteoma frontal foi descoberto pela investigação tomográfica devido ao quadro de epistaxe bilateral anterior recidivante.

Os antecedentes, sintomatologia, localização e volume das lesões estão mostrado na Tabela 1. Os exames complementares realizados em todos os pacientes foram radiografia simples (RX) e tomografia computadorizada (TC) de seios paranasais com cortes axiais e coronais (Figs 1 a 3 ). As dimensões do osteoma foram medidas na TC de crânio. Os osteomas volumosos foram considerados como tendo mais de 3 centímetros de diâmetro na sua maior dimensão.

Tabela 1. Série de pacientes operados de osteoma de seio frontal.

\begin{tabular}{|c|c|c|c|c|c|c|c|}
\hline Paciente & Sexo & Idade & Antecedentes & Sintomas & $\begin{array}{l}\text { Localização } \\
\text { anatômica }\end{array}$ & Volume & $\begin{array}{l}\text { Técnica } \\
\text { cirúrgica }\end{array}$ \\
\hline RS & $M$ & 12 & Sinusite & Cefaléia & $\begin{array}{l}\text { Lateromedial } \\
\text { Direita }\end{array}$ & $<3 \mathrm{~cm}$ & Coronal \\
\hline $\mathrm{MN}$ & $M$ & 32 & Sinusite & Cefaléia & $\begin{array}{l}\text { Medial } \\
\text { Direita }\end{array}$ & $<3 \mathrm{~cm}$ & Coronal \\
\hline AL & $\mathrm{M}$ & 47 & Calvície & Cefaléia & $\begin{array}{l}\text { Medial } \\
\text { Direita }\end{array}$ & $>3 \mathrm{~cm}$ & Supraciliar \\
\hline IV & $\mathrm{F}$ & 55 & - & Cefaléia & $\begin{array}{l}\text { Lateral } \\
\text { Direita }\end{array}$ & $<3 \mathrm{~cm}$ & Coronal \\
\hline SA & $\mathrm{F}$ & 46 & Epistaxe & $\begin{array}{l}\text { Obstrução } \\
\text { Nasal }\end{array}$ & $\begin{array}{l}\text { Seio Frontal } \\
\text { Direito }\end{array}$ & $>3 \mathrm{~cm}$ & Coronal \\
\hline
\end{tabular}

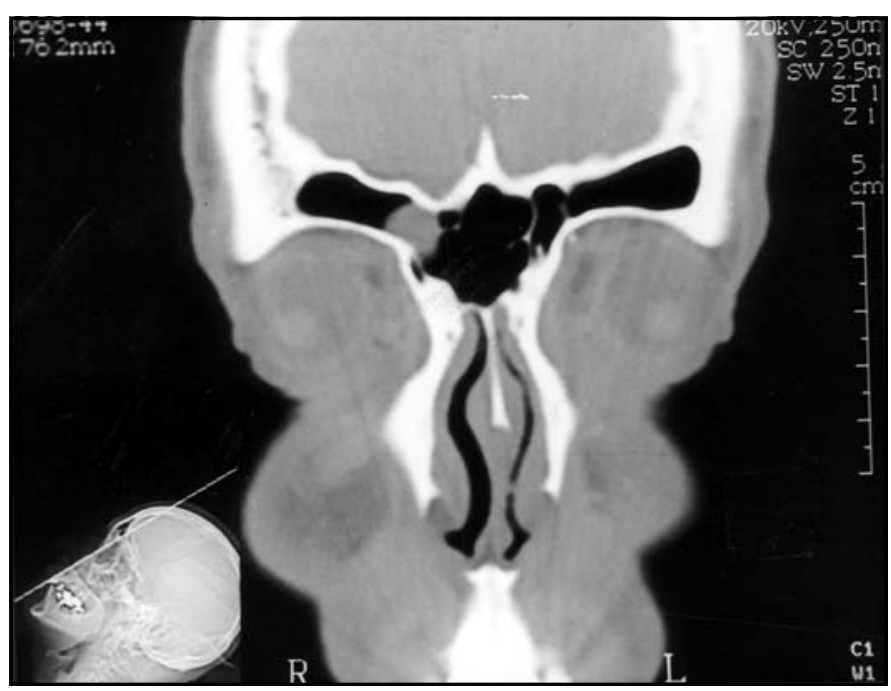

Fig 1. TC em corte axial evidenciando osteoma em seio frontal direito (Paciente MN).

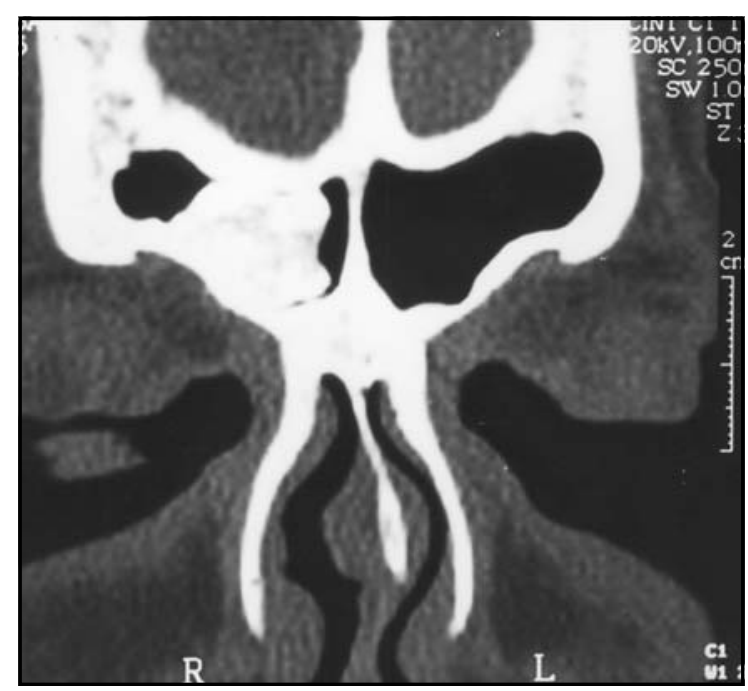

Fig 2. TC em corte axial evidenciando osteoma de seio frontal com extensão para seio etmoidal (Paciente AL). 


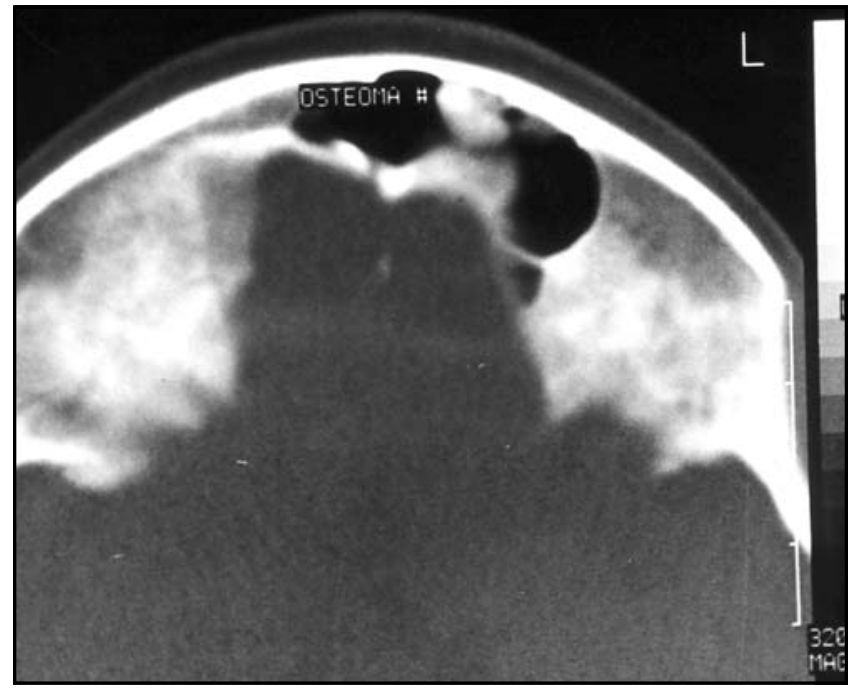

Fig 3. TC em corte axial pré-operatória com osteoma frontal causando divisão do seio (Paciente RS).

O tratamento consistiu na ressecção cirúrgica do osteoma via coronal com retalho de osso frontal limitado em quatro pacientes, e abordagem superciliar em um paciente que apresentava calvície. A decisão da técnica cirúrgica entre coronal e supraciliar foi estética, reservando-se a abordagem supraciliar para um paciente com calvície, apesar do tumor ser volumoso com extensão para seio etmoidal. Nenhuma dificuldade técnica intra-operatória foi atribuída à escolha da abordagem. O óstio naso-frontal não foi obstruído no intra-operatório. Nenhum paciente submetido à abordagem via coronal apresentou sangramento superior a $500 \mathrm{ml}$. O resultado pós-operatório anatômico (Fig 4) e estético foi satisfatório em todos.

\section{DISCUSSÃO}

Osteoma pode ser observado em qualquer faixa etária, sendo mais prevalente nas terceira e quarta décadas. Há preponderância no sexo masculino, variando de $2: 1$ a 3,75:1 ${ }^{1,4}$. A idade média de 38,4 anos e a preponderância do sexo masculino de 3:2 em nossa série é similar a literatura ${ }^{1,4}$.

A etiologia dos osteomas é desconhecida, admitindo-se teorias traumática, embrionária, infecciosa e genética. Antecedente traumático é encontrado em percentagem variável de $5 \%$ a $29 \% 4,9$. A teoria embrionária é baseada na hipótese de que os tumores podem se desenvolver sobre a junção de dois tecidos embriologicamente diferentes. O osteoma se desenvolveria sobre a junção do osso frontal de origem membranosa e do osso etmoidal de origem endocondral ${ }^{3,7}$. A etiologia embrionária como causa isolada dos osteomas do seio frontal é pouco provável, pois a localização destes tumores na junção des-

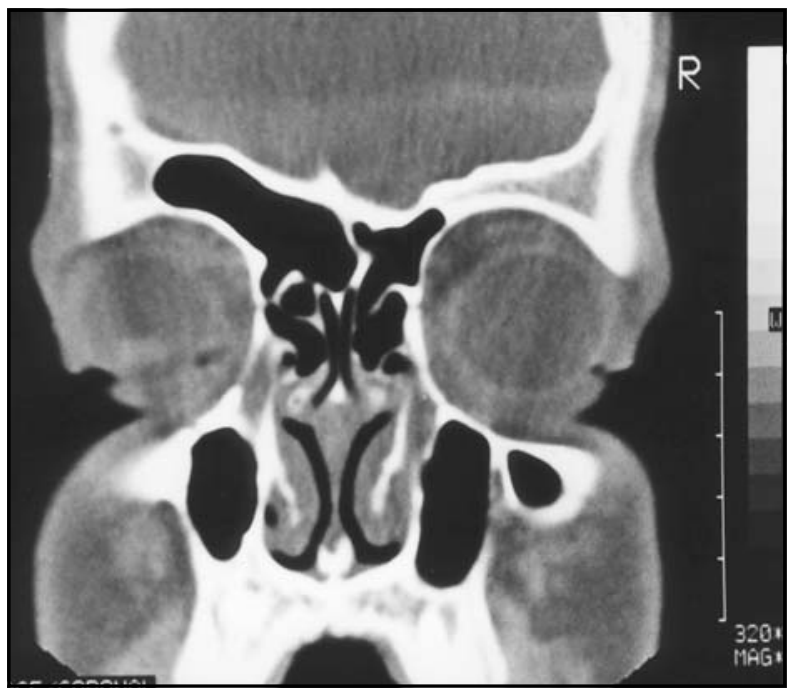

Fig 4. TC em corte coronal pós-operatório com remoção total do osteoma e preservação anatômica do óstio nasofrontal (Paciente RS).

tes ossos é encontrada em apenas $10 \%$ a $20 \%$ dos pacientes ${ }^{4}$. Encontramos em $30 \%$ dos pacientes antecedentes de infecção sinusal ${ }^{3}$. As alterações infecciosas crônicas da mucosa sinusal como causa do osteoma ${ }^{4,9}$ são altamente questionáveis, podendo ser consequência do osteoma e não a sua causa ${ }^{4}$. Admite-se a possibilidade da participação de um padrão genético de penetrância, expressividade e dominância desconhecidos pela ocorrência de osteomas múltiplos na síndrome autossômica dominante de Gardner, na comunidade mórmon de $U \operatorname{tah}^{3,6}$. Nenhum dos nossos pacientes apresentou história prévia de trauma, sendo sinusite prévia relatada em dois, e nestes sem qualquer correlação causal direta, já que eram maxilares.

Em aproximadamente $70 \%$ dos pacientes encontramos dor facial, frontal ou cefaléia, esta última com localizações variáveis e sem explicação fisiopatológica apropriada 4 . Encontramos antecedentes de cefaléia em quatro pacientes de nossa série, ou seja em $80 \%$. O osteoma não pode ser considerado como agente causador da cefaléia, admitindo-se outras etiologias. A cefaléia deve ser considerada como a queixa que motivou a realização de um exame radiológico complementar que forneceu o diagnóstico do osteoma. Pode existir evidência de mucocele ou sinusite usualmente associada ao bloqueio dos ductos nasais ${ }^{7}$. Os sinais decorrentes do aumento do osteoma não são frequentes. Os sintomas compressivos, quando ocorrem, estão relacionados com a direção do crescimento. Podemos encontrar deformidade craniana quando o osteoma cresce an- 
teriormente em $10 \%$ a $38 \%$ dos pacientes 4 . Proptose, diplopia, exoftalmia, síndrome de Brown adquirida, amaurose fugaz e diminuição da acuidade visual em paciente com extensão inferior do tumor; abscesso cerebral, fístula liquórica e pneumoencéfalo com manifestações centrais de demência, meningites, crise convulsiva e hemiparesia podem ocorrer no caso de extensão posterior do tumor ${ }^{3,1}$. Sinusite independente da sua localização é encontrada em aproximadamente $22 \%$ dos casos $^{5}$. Mucocele e piocele ocorrem em $12,5 \%$ a $50 \%{ }^{2}$.

O diagnóstico por imagem é obtido pela radiografia simples. A avaliação por TC de seios paranasais com janela óssea e reconstrução tridimensional é útil no planejamento cirúrgico, com a identificação do óstio nasofrontal. A TC permite avaliar a localização, local de implantação e a expansão da lesão, seu volume e eventuais lesões associadas. Dois pacientes apresentavam osteomas volumosos com diâmetro maior de $3 \mathrm{~cm}$. A localização no seio frontal direito em todos os pacientes foi considerada como fortuita.

Os osteomas frontais possuem aspecto radiológico indistinto das outras lesões fibro-ósseas que incluem osteoma fibroso, displasia fibrosa e fibromas ossificados $^{7}$. O osteoma fibroso apresenta estroma fibroso mais proeminente que o separa do trabeculado ósseo espessado. O osteoma fibroso ocorre na mesma faixa etária e possui o mesmo comportamento benigno que os osteomas típicos. A displasia fibrosa apresenta aspecto radiológico de menor definição e irregularidade. O fibroma ossificante apresenta evolução clínica mais agressiva. A forma psamomatosa do osteoma ossificante incide em crianças menores, no seio etmoidal, com comportamento biológico localmente maligno ${ }^{7}$. A TC apresenta vantagens significativas em relação à ressonância magnética para a avaliação óssea, podendo mesmo identificar áreas líticas ósseas, com diagnóstico de patologias outras do seio, que podem ter uma extensão intracraniana como mucocele. A mucocele demonstra uma imagem de lesão densa sem captação de contraste, o diagnóstico sendo de mucopiocele se existir captação de contraste ${ }^{2}$. Mapeamento ósseo por radio-fármacos pode ser empregado podendo demonstrar um metabolismo aumentado. A captação do osteoma demonstra grande potencial de crescimento sendo por si indicativo de cirurgia. No caso de um teste negativo, traduz um osteoma inerte com possibilidade de seguimento clínico'.

Existem dois tipos histológicos de osteomas frontais: osteoma ebúrneo que é composto por osso denso, endurecido que contém apenas quantidade mínima de tecido fibroso; e o osteoma esponjoso que contem osso esponjoso, podendo ser encontrado na periferia do osteoma ebúrneo. Uma terceira forma pode ser encontrada, tendo o padrão histológico de uma combinação dos dois tipos descritos previamente ${ }^{7,3}$.

Os osteomas são histologicamente benignos, bem localizados, com tendência pequena de recidiva após ressecção adequada ${ }^{3}$. O diagnóstico diferencial histológico inclui osteoma fibroso, displasia fibrosa, fibroma ossificante, osteoblastoma, tumor de células gigantes, osteossarcoma, metástase, mucocele, meningeoma calcificado intra-sinusal, hematomas e pó$\operatorname{lipos}^{3}$. Na nossa série todos os pacientes apresentavam a forma ebúrnea.

A abordagem cirúrgica nos osteomas frontais pode ser transfacial ou coronal. A transfacial pode ser realizada mediante incisão cutânea supraciliar ou paralateronasal. A transcraniana ou coronal, com incisão de couro cabeludo coronal ou bitragus, pode ser preferível à transfacial, pois oferece uma exposição mais apropriada do seio frontal ${ }^{4,10,1}$. A abertura e rebatimento bilateral do osso frontal também permitem a inspeção intracraniana, da órbita, e um reparo da dura máter caso necessário, levando a perda sanguínea variável entre 225 e $650 \mathrm{ml}^{4,1}$. A abordagem supraciliar ou paralateronasal apresenta sangramento em média 50\% menor que a coronal, menor exposição do campo operatório, menor edema de face e expectativa de um período de internação hospitalar pós-operatória menor. Nos pacientes calvos a abordagem supraciliar ou posterolateronasal deve ser empregada em decorrência do resultado estético final ${ }^{3,4}$.

Após a abertura ampla da fossa anterior devemos identificar o osteoma correlacionando-o com a TC. Os osteomas medianos com extensão para os seios etmoidal e esfenoidal possuem aderência acentuada apenas na sua implantação, podendo ser removido totalmente sem dificuldade ${ }^{10}$. A remoção do osteoma pode ser feita com formão ou broca com motor de alta frequência ${ }^{4}$. Os osteomas laterais que têm origem no teto da órbita e invadem a cavidade orbitária necessitam de remoção do teto orbitário com o objetivo de descomprimir a cápsula orbitária. A remoção deve se estender posteriormente ao canal óptico quando o tumor causa compressão do nervo óptico ${ }^{10}$. Podemos empregar materiais biológicos, osso autólogo e materiais sintéticos na correção de falhas estéticas em ressecção de osteomas volumosos ou infiltrativos ${ }^{10}$. Osteomas volumosos 
podem ser removidos por craniotomia bifrontal ampla com reconstrução associada, tendo-se acesso à órbita bilateralmente ${ }^{10}$. Não realizamos a oclusão do ducto nasofrontal ${ }^{4}$, apesar de preconizado por alguns autores ${ }^{1,3,4,6}$. A oclusão não foi realizada por considerarmos este procedimento desnecessário na profilaxia de sinusopatias. No seguimento pós-operatório nenhum paciente apresentou sinusopatia.

Pode ser empregado laser de gás carbônico nos osteomas pequenos ${ }^{3}$ e também cirurgia endoscópica endonasal ${ }^{10}$. A cirurgia endoscópica endonasal apresenta como vantagem a possibilidade de remoção da lesão sem a necessidade de incisão cutânea, podendo ser adequadamente empregada nos osteomas menores e médios. Nos osteomas volumosos, invasivos e de localização lateral, apesar de factível, existe a possibilidade de maior taxa de recidiva com o emprego da técnica endoscópica.

O resultado estético foi considerado satisfatório em todos os pacientes, não existindo desnível a ósseo ou cicatrização inadequada. Não existiu qualquer dificuldade técnica na cirurgia do paciente calvo que foi submetido a abordagem supraciliar. A indicação desta abordagem pode ser ampliada para os pacientes não calvos. As complicações pós-operatórias são infrequentes e incluem pneumatocele extradural, abscesso extradural, fístula liquórica, me- ningite ${ }^{10}$, alopécia pela incisão coronal, paralisia temporária do ramo frontal do nervo facial ${ }^{1}$, deformidade do frontal, anestesia cutânea e neuralgia do nervo supraorbitário ${ }^{11}$. Não observamos complicações em nossos pacientes.

\section{BIBLIOGRAFIA}

1. Chen PK-T, Chen Y-R, Chang C-N. Treatment of frontal sinus osteoma using a craniofacial approach. Ann Plast Surg 1997;38:455-459.

2. Shady JA, Bland LI, Kazee AM, Plcher WH. Osteoma of the frontoethmoidal sinus with secondary brain abscess and intracranial mucocele: case report. Neurosurgery 1994;34:920-923.

3. Rappaport JM, Attia EL. Pneumoencephalus in frontal sinus osteoma: case report. Otolaryngol 1994;23:430-435.

4. Detsouli M, Laraqui NZ, Benghalem A, et al. L'ostéome du sinus frontal : a propos de 10 cas. Ann Otolaryngol Chir Cervicofac 1995;112:293297.

5. Smith ME, Calcaterra TC. Frontal sinus osteoma. Ann Otol Rhinol Laryngol 1989;98:896-900.

6. Hehar SS, Jones NS. Fronto-ethmoid osteoma: the place of surgery. J Laryngol Otol 1997;111:372-375.

7. Chen PK, Chen Y, Chang C. Frontal sinus osteoma. Ann Plast Surg 1977;38:455-459.

8. Savic DL, Djeric DR. Indications for the surgical treatment of osteomas of the frontal and ethmoid sinuses. Clin Otolaryngol 1990;15:397-404.

9. Coste A, Cheavlier E, Beautru R, Alsamad IA, Salvan D, Peynegre R. Ostéomes des cavités naso-sinusiennes: indications opératoires et place de la chirurgie endonasale endoscopique. Ann Otolaryngol Chir Cervicofac 1996;113:197-201.

10. Maiuri F, Iaconetta G, Giamundo A, Stella L, Lamaida. Fronto-etmoidal and orbital osteomas with intracranial extension: report of 2 cases. J Neurosurg Sci 1996;40:65-70.

11. Mendizabal JMB, Martinez CP, Vidal AM. Colgajo osteoplastico del seno frontal: estudio de 47 casos. Acta Otorrinolaringol Esp 1998; 49:380-384. 